\title{
A New Computerised Method of Multiple Labelling Detection and Particle Evaluation
}

\author{
L.H. Monteiro-Leal, ${ }^{*}$ H. Tröster, ${ }^{* *}$ L. Campanati, ${ }^{* * *}$ H. Spring, ${ }^{* *}$ and M. Trendelenburg ${ }^{* *}$ \\ * Universidade do Estado do Rio de Janeiro-IB-DHE-Lab. de Microscopia e Processamento de \\ Imagens-Av. Prof. Manoel de Abreu 48, Maracanã, 20550-170, Rio de Janeiro, Brazil \\ **Biomedical Structure Analysis (A0601), DKFZ, INF 280, D-69120 Heidelberg, Germany \\ **** UFRJ- Lab. de Ultraestrutura Celular Hertha Meyer. Inst.de Biofísica Bl-G subsolo, Brazil
}

In this work a new computerized method of multiple labelling detection, identification and evaluation of gold particles in EM (electron microscopic) sections is presented. After antigen localisation by immunofluorescence, EM embedding and the simultaneous application of two different gold-coupled antibodies, specimens were analysed in a LEO EM 912 Omega (LEO Electron Microscopy, Oberkochen, Germany) equipped with a $2 \mathrm{~K}$ x $2 \mathrm{~K}$ pixel Slow-Scan CCD camera (Proscan elektronische Systeme, Scheuring, Germany) connected to a computer with analySIS 3.1 (Soft Imaging System, Münster, Germany) image processing software. Automated gold particle identification and counting was done as follows (FIG. 1): A - Acquisition of a high resolution (2048 pixel $^{2}$ ), high dynamic range-image after adjustment of contrast and brightness. B- Definition of an ROI (region of interest) and selection of a threshold window (gap between B and $\mathrm{C}$ and in FIG. 2), C-creating a binary image. This image and the localised objects underwent a classification routine, D- resulting in the identification, counting and overlay of the different particle types in the image.

The decisive step is the acquisition of a high D.R. image, that will be digitised and used by the software as a 16 bit image (65536 grey scales). The next step is the classification. The system recognises different particle sizes and shapes. Several modules were designed to choose among three size differentiation routines: $3 / 10 \mathrm{~nm}, 6 / 15 \mathrm{~nm}, 10 / 25 \mathrm{~nm}$. After the selection of the pair used, the computer classifies the particles by first identifying the single particles within both a specific size- and a spherical symmetry-range, and then, for the small particles, the ones that were clustered. In this latter case the particle number is calculated depending on the cluster size. The result is the distinct evaluation of each of the two particle types used, including the particle numbers and coloured presentation. The usefulness of the method became obvious when it was associated to automatic image montage utilities. As shown in FIG. 3 (A), analySIS 3.1 allows an assembly of a wide field image (in this case a protozoon - Giardia lamblia). Even in large areas the reliable particle detection, counting, determination of the total area analysed, and the presentation of the differential distributions of the gold granules identifying specific antigens (tubulin: $6 \mathrm{~nm}$, in green; actin: $15 \mathrm{~nm}$, in red) were possible. For a better control of the detection reliability, FIG. 3B shows a zoomed high-contrast region of the cell before, and FIG. 3C the same region after the application of the method.

\section{References}

[1] This study was supported by SR-2 UERJ, Deutscher Akademischer Austauschdienst (DAAD) and by the grant No.10944A by way of agreement between the German Ministry of Science and Education (BMBF) and the LEO Electron Microscopy GmbH (Oberkochen, Germany). 


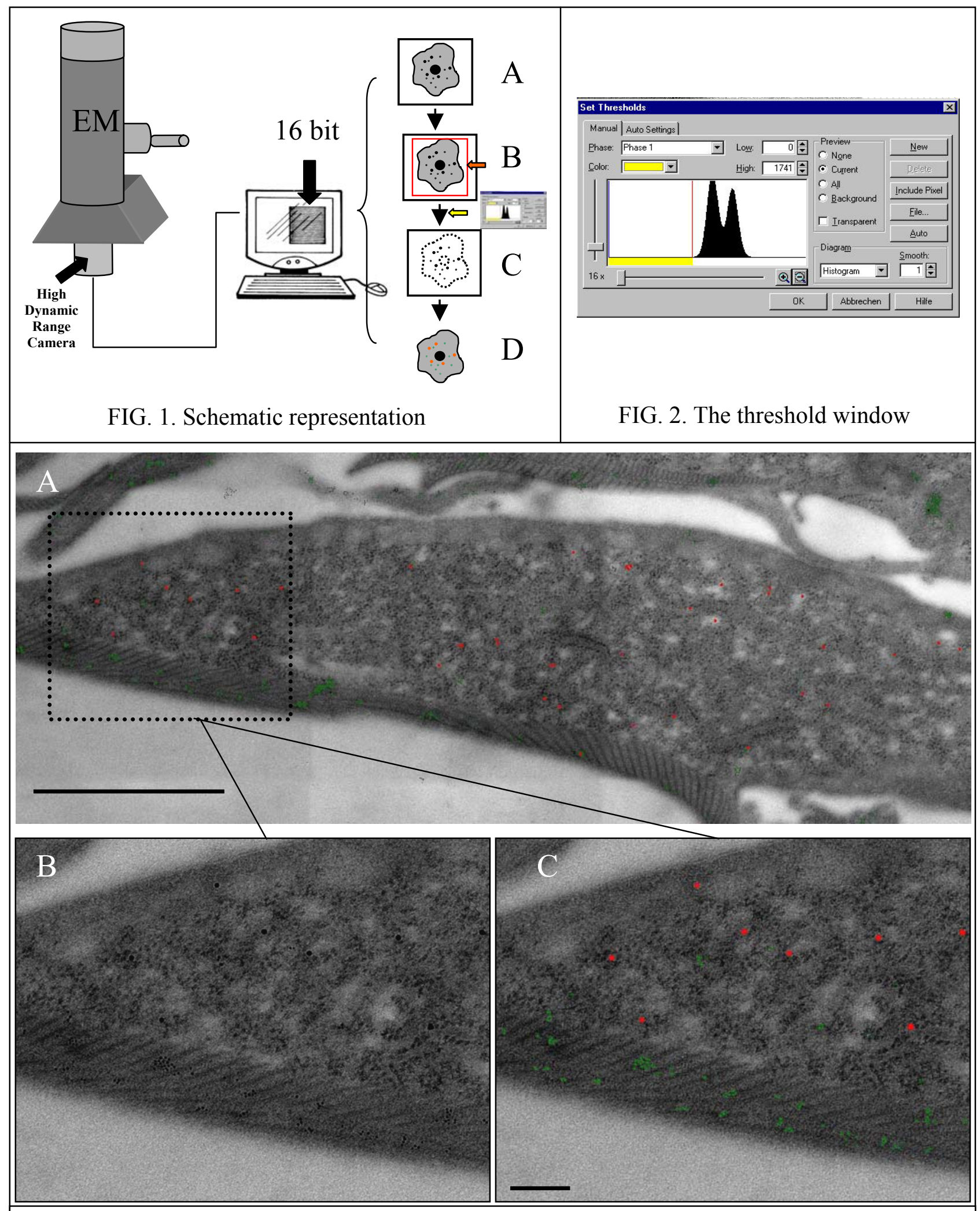

FIG. 3. View of a uranyl acetate (5\%, water)-contrasted LRWhite section after image montage (A) and particle evaluation by the module (green: tubulin $/ 6 \mathrm{~nm}$; red: actin/15 nm). Zoomed region: details before $(B)$ and after $(C)$ the particle detection. Bars: $0.6 \mu \mathrm{m}(\mathrm{A}) ; 0.1 \mu \mathrm{m}(\mathrm{B}, \mathrm{C})$. 